\title{
Some Properties of Topology Fuzzy Modular Space
}

Noori F. Al-Mayahi ${ }^{1}$ and Al-ham S . Nief ${ }^{2}$

Department of Mathematics College of Computer Science and Information Technology

University of AL -Qadisiyah ,Diwanyah -Iraq

E-mail: nafm60@yahoo.com

Recived : $27 \backslash 1 \backslash 2019$

Revised : $12 \backslash 2 \backslash 2019$

Available online : $24 / 4 / 2019$

\begin{abstract}
:
In the present paper, the authors have introduced and studied fuzzy modular space. they have investigated some properties of this space in the open and closed balls. Also the authors discussed the convex set and the locally convex in fuzzy modular space. The result obtained are correct and the methods used are interesting.
\end{abstract}

M.S.C: 03E72, 46A80 .

Keywords: fuzzy set, modular space, fuzzy modular space. 


\section{Introduction}

The concept of fuzzy sets was introduced by

Zadeh [5] in 1965 and there after several authors

applied it to different branches of pure and applied

mathematics. The concept of modular space was

introduction by S.S. Abed, K .A .Abdul Sada in

2017.The concept of fuzzy modular space was

introduced by Young Shen and Wei Chen [7] in

2013.

\section{Definition(1.1) [4]}

A fuzzy set $A$ in $X$ (or a fuzzy subset in $X$ )

is a function from $X$ into $I=[0,1]$ that is $A \in I^{x}$

$A \in I^{x}$.

De Definition(1.2)[6] Let $X$ be a linear space.

over $F$. A function $M: X \rightarrow[0, \infty]$ is called modular if:

1. $M(x)=0 \Leftrightarrow x=0$,

2. $M(\alpha x)=M(x)$ for $\alpha \in F$ with $|\alpha|=1$

for all $\alpha \in F$.

3. $M(\alpha x+\beta y) \leq M(x)+M(y)$

iff $\alpha, \beta \geq 0$,for all $x \in X$.

\section{Definition (1.3)[2]}

A binary operation $*:[0,1] \times[0,1] \rightarrow[0,1]$

is called a continuous t-norm if it satisfies the following

1. * is commutative and associative .

2. $*$ is continuous .

2. $a * b \leq c * d$ whenever $a \leq c, b \leq d$ and

$a, b, c, d \in[0,1]$.

Three common examples of the continuous
Noori .F/Al-ham .S

$t-$ norm are

1. $a *_{\mathrm{M}} b=\min [a, b]$.

2. $a *_{p} b=a . b$.

3. $a *_{\mathrm{L}} b=\operatorname{Max}\{a+b-1,0\}$.

Lemma (1.4): [2]

If the $t$-norm $*$ is continuous , then

1. for every $\gamma_{1}, \gamma_{2} \in(0,1)$ with $\gamma_{1}>\gamma_{2}$, there exist $\gamma_{3} \in(0,1)$ such that $\gamma_{1} * \gamma_{3} \geq \gamma_{2}$.

2. for every $\gamma_{4} \in(0,1)$, there exist $\gamma_{5} \in(0,1)$

such that $\gamma_{5} * \gamma_{5} \geq \gamma_{4}$.

\section{Definition (1. 5): [7]}

A fuzzy modular space is an ordered triple $(X, \mu, *)$ such that $X$ is a vector space, $*$ is continuous t- norm and $\mu$ is a fuzzy set on $X \times(0, \infty)$ satisfying the following condition, for all $x, y \in X$ and $\alpha, \beta \geq 0$ with $\alpha+\beta=1$ :

1. $\mu(x, t)>0$.

2. $\mu(x, t)=1$ for all $t>0$ if and only if $x=0$.

3. $\mu(x, t)=\mu(-x, t)$.

4. $\mu(\alpha x+\beta y, s+t) \geq \mu(x, s) * \mu(y, t)$.

5. $\mu(x,):.(0, \infty) \rightarrow(0,1]$ is continuous.

Generally, if $(X, \mu, *)$ is fuzzy modular space, we say that $(\mu, *)$ is a fuzzy modular on $\mathrm{X}$. Moreover, the triple $(X, \mu, *)$ is called $\beta$-homogeneous if every $x \in X, \mathrm{t}>0$ and $\lambda \in \mathbb{R} \backslash\{0\}$ $\mu(\lambda x, t)=\mu\left(x, \frac{t}{|\lambda|^{\beta}}\right), \quad$ where $\beta \in(0,1]$.

\section{Example (1.6): [7]}

Let $X$ be a vector space and let $\rho$ be a modular on $X$. Take t-norm $a * b=a *_{M} b$. For every $t \in(0, \infty)$ define $\mu(x, t)=t /(t+p(x))$ 
Noori .F/Al-ham .S

$\begin{array}{ll}\text { for all } x \in X \text {. Then }(X, \mu, *) \text { is a fuzzy modular } & 1 \\ \text { space. } & 2 \\ \text { Remark (1.7): [7] } & 1 \\ & \end{array}$

Note the above conclusion still holds even if

The $\mathrm{t}$-norm is replaced by $a * b=a *_{p} b$ and $a * b=a *_{L} b$, respectively.

Example (1.8): [7]

Let $X=\mathbb{R}$, take t-norm a $* b=a *_{M} b$. For every $x, y \in X$ and $t \in(0, \infty)$, we define If we take $\alpha=\sqrt{2} / 2, \beta=1-\frac{\sqrt{2}}{2}, x \neq y$, and Proof :

$x, y \in Z$, then we know $\alpha x+\beta y \in \mathbb{R} \backslash Z$.Thus, for allt, $s>0$, we have $\mu(\alpha x+\beta y, t+s)=1 / 4$.

But $\mu(x, t) *_{\mathrm{M}} \mu(y, s)=\min \{\mu(x, t), \mu(y, s)\}=1 / 2$. $\left(\mu, *_{\mathrm{M}}\right)$ is not a fuzzy modular on $X$

\section{Example (1.9)[7]}

Let $X=\mathbb{R}, \rho$ is amodular on $X$,which is defined by $\rho(x)=|x|^{\beta}$, where $\beta \in(0,1]$.Take t-norm $a * b=a *_{p} b$. for every $t \in(0, \infty)$, we define $\mu(x, t)=\frac{1}{e^{\rho(x) / t}}$

for all $x \in X$.Then $(X, \mu, *)$ is $\beta$-homogenous fuzzy modular space.

\section{Definition (1.10) : [1]}

A fuzzy metric space is an ordered triple $(X, M, *)$ such that $X$ is a nonempty set, $*$ is a continuous $\mathrm{t}-$ norm , and $M$ is a fuzzy set on $X \times X \times(0, \infty)$ satisfying the following conditions :
1. 0 . 2. 1 if and only if $x=y$. 3. $M(y, x, t)$.

4. $M(y, z, t) \leq M(x, z . t+s)$.

5. $M(x, y, \cdot):(0, \infty) \rightarrow(0,1]$ is continuous .

\section{Theorem (1.11):}

Every fuzzy modular space is fuzzy metric space .

Let $(X, \mu, *)$ be a fuzzy modular space, defined $M: X \times X \times(0, \infty) \rightarrow[0,1]$ as follows: $M(x, y, t)=\mu(x-y, t) \quad \forall x, y \in X$

1. Let $x, y \in X$

$\stackrel{\text { Obviously }}{\Rightarrow} \underset{x}{ }-y \in X \Rightarrow \mu(x-y, t)>0 \Rightarrow M(x, y, t)>0$

2. uppose $M(x-y, t)=1 \Leftrightarrow 1=M(x, y, t)$ $=\mu(x-y, t) \Leftrightarrow x-y=0 \Leftrightarrow x=y$

3. Let $x, y \in X$ and $t>0$

$M(x, y, t)=\mu(x-y, t)=\mu(y-x, t)$

4.

4. Let $x, y, z \in X$ and $s, t>0$

$$
\begin{gathered}
M(x, y, t) * \mu(y, z, t)=\mu(x-y, t) * \mu(y-z, s) \\
\leq \mu((x-y)+(y-z), t+s)=\mu(x-z, t+s) \\
=M(x, z, t+s)
\end{gathered}
$$

$5 . M(x, y, \cdot)=\mu(x-y, \cdot):(0, \infty) \rightarrow[0,1]$ is continuous.

4

$\operatorname{Then}(X, M, *)$ is fuzzy metric space .

Theorem (1. 12): [7]

If $(X, \mu, *)$ is a fuzzy modular space, 
Noori .F/Al-ham .S

then $\mu(x,$.$) is nondecreasing for all x \in X$.

\section{Definition (1.13): [7]}

Let $(X, \mu, *)$ be a fuzzy modular space .we define the open ball $B(x, r, t)$ and the closed ball

$B[x, r, t]$ with center $x \in X$ and radius

$0<r<1$ as follows: For $t>0$

$B(x, r, t)=\{y \in X: \mu(x-y, t)>1-r\}$ open balls

$B[x, r, t]=\{y \in X: \mu(x-y, t) \geq 1-r\}$ closed balls

\section{Theorem (1: 14)}

If $(x, \mu, *)$ is a $\beta$-homogenous fuzzy modular space then $B(x, r, t) \subset B\left(x, r, \frac{t}{2}\right)$.

\section{Proof:}

Let $B\left(x, r_{11}, \mathrm{t}\right)$ and $B\left(x, r_{2}, \mathrm{t}\right)$ be open balls with the same center $x \in X$ and $t>0$ with the radius $0<r_{1}<1$ and $0<r_{2}<1$, respectively.

Then we either have :

$B\left(x, r_{1}, t\right) \subset B\left(x, r_{2}, \mathrm{t}\right)$ or $B\left(x, r_{2}, \mathrm{t}\right) \subset B\left(x, r_{1}, \mathrm{t}\right)$.

Let $x \in X$ and $t>0$.consider the open ball

$B\left(x, r_{1}, t\right)$ and $B\left(x, r_{2}, t\right)$ with $0<r_{1}<1$,

$0<r_{2}<1$.If $r_{1}=r_{2}$, then the Theorem holds.

Next, we assume that $r_{1} \neq r_{2}$. we may

Assume without loss of generality that

$0<r_{1}<r_{2}<1$

Now let $y \in B\left(x, r_{1}, t\right) \Rightarrow \mu(x-y, t)<r_{1}<r_{2}$

Hence $y \in B\left(x, r_{2}, t\right)$. This shows that

$B\left(x, r_{1}, t\right) \subset B\left(x, r_{2}, t\right)$. By assuming that

$0<r_{2}<r_{1}<1$, we can similary to show

$B\left(x, r_{2}, t\right) \subset B\left(x, r_{1}, t\right)$.

Definition (1.15):
Theorem (1.16): [7]

Let $(X, \mu, *)$ be a $\beta$-homogenous fuzzy modular space .Every $\mu$.ball $B(x, r, t)$ in $(X, \mu, *)$ is a $\mu$-open set.

\section{Theorem (1.17):}

The intersection number of open sets in fuzzy modular space is open sets

\section{Proof:}

Let $(X, \mu, *)$ be a fuzzy modular space and let $\left\{G_{i}: i=1,2, \ldots \mathrm{m}\right\}$ be a finite collection of open set in the fuzzy modular space. Let

$$
\mathrm{H}=\cap\left\{G_{i}, i=1,2, \ldots, m\right\}
$$

To prove That $\mathrm{H}$ is an open set let $x \in H \Rightarrow x \in G_{i} \quad \forall_{i}=1,2, \ldots \mathrm{m}$

|Since $G_{i}$ open set $: \forall_{i} \Rightarrow \exists r_{i} \in(0,1)$ and

$$
\begin{gathered}
t_{i}>0>\ni B\left(x, r_{i}, t_{i}\right) \subset G_{i} \\
\text { Let } t_{k}=\max \left\{t_{1}, t_{2}, \ldots, t_{n}\right\} \text { and } \\
r_{k}=\min \left\{r_{1}, r_{2}, \ldots, r_{n}\right\} \\
\Rightarrow B\left(x, r_{k}, t_{k}\right\} \subset G_{i} \\
\Rightarrow B\left(x, r_{k}, t_{k}\right) \subset \cap G_{i} \Rightarrow B\left(x, r_{k}, t_{k}\right) \subset H
\end{gathered}
$$

Then $H$ is open set

Theorem (1.18) The union of an arbitrary collection of open set in fuzzy modular space are open sets

\section{Proof:}

Let $(X, \mu, *)$ be a fuzzy modular space and 
Noori .F/Al-ham .S

let $\left\{\gamma_{\lambda}: \lambda \in \Lambda\right\}$ be an arbitrary collection of open sets in $X$. Let $G=\cup\left\{\gamma_{\lambda}: \lambda \in \mathcal{\Lambda}\right\}$ We must to prove $G$ is open

Let $X \in G \Rightarrow X \in \gamma_{\lambda}$ for some $\lambda \in \wedge$

Since $\gamma_{\lambda}$ is open set

$\Rightarrow$ there exist $r \in(0,1)$ such that $B(x, r, t) \subset$

$\gamma_{\lambda}$

Since $\gamma_{\lambda} \subset G \Rightarrow$ Then $B(x, r, t) \subset G$

$\Rightarrow G$ is open set .

\section{Theorem (1.19):}

Let $(X, \mu, *)$ be afuzzy modular space

if $C$ and $D$ are open sets in a vector space $X$ then $C+D$ is open set in $X$.

\section{Proof:}

Let $x \in X$ and a $\in C$, since $A$ is open set then

there exist $r \in(0,1)$ such that

$B(a, r, t) \subset C$,

then $B(a, r, t)+x \subset C+x$

$\Rightarrow B(a+x, r, t) \subset C+x \Rightarrow C+x$

is open set in $X$ for all $x \in X$

Since $C+D=U\{C+d: d \in D\}$

Then $C+D$ is open set in $X$.

\section{Theorem (1.20):}

Every single set in fuzzy modular space is closed set .

\section{Proof:}

Let $X$ be a fuzzy modular space

Let $B=\{x\}$ be a set in $X$, to prove $B$ is closed set

Let $y \in A^{c} \Rightarrow y \neq X$ $\mu(y-x, t)>0($ since $X$ is fuzzy modular space $)$

$$
\begin{aligned}
& X \notin B(y, r, t)=\{a \in X: \mu(a-y, t)>1-r\} \\
& B \cap B(y, r, t)=\emptyset \Longrightarrow B(y, r, t) \subseteq A^{c}
\end{aligned}
$$

Then $y \in B(y, r, t) \subseteq B^{c} \Rightarrow y$ is interior point

Then $B^{c}$ is open set

Then $B$ is closed set .

Corollary (1.21) Every finite set in fuzzy

modular space is closed set.

\section{Definition (1.22):}

A subset $U$ of $X$ is said to be a neighborhood of $x \in X$ in $(X, \mu, *)$ if there exist $r \in(0,1)$ and $t \in(0, \infty)$ such that $B(x, r, t) \subset U$.

Definition (1.23):[2]

A subset $A$ of a vector space $X$ over $F$ is called convex set if $\alpha A+(1-\alpha) A \subset A$ for all $0 \leq \alpha \leq 1$.

\section{Theorem (1. 24):}

Every open and closed balls in fuzzy modular space are convex sets.

\section{Proof:}

Let $y_{1}, y_{2} \in B(x, r, t)$ such that $r \in(0,1), t>0$, $\mu\left(x-y_{1}, t\right)>1-r$ and $\mu\left(x-y_{2}, t\right)>1-r$. Now ,we have to prove $\alpha y_{1}+(1-\alpha) y_{2} \in B(x, r, t)$

$$
\begin{aligned}
& \mu\left(x-\left(\alpha y_{1}+(1-\alpha) y_{2}, t\right)\right. \\
= & \mu\left(x-\alpha y_{1}+(1-\alpha) x-(1-\alpha) y_{2}, t\right) \\
& =\mu\left(\alpha\left(x-y_{1}\right), t\right)+\mu\left((1-\alpha)\left(x-y_{2}\right), t\right) \\
= & \mu\left(x-y_{1}, t\right) * \mu\left(x-y_{2}, t\right) \\
> & 1-r * 1-r-r \\
= & 1-r
\end{aligned}
$$


Then $B(x, r, t)$ is convex set. Then $B\left(x, \frac{1}{n}, t\right)$ contains $x_{\mathrm{n}} \in A$ and similary, we can prove $B[x, r, t]$ is convex set. since $\lim _{n \rightarrow \infty} \mu\left(x_{n}-x, t\right)=1$

Theorem (1.25):

Conversely :Suppose the condition is true ,to

Let $X$ be a vector space . if $A$ is convex $\operatorname{prove} \operatorname{lin}^{\prime}$

fuzzy modular space then $\bar{A}$ is confl $\operatorname{x}_{\chi_{n}} \operatorname{set}$.in $A$ and $x_{n} \rightarrow x$ then $x \in A$

proof :

Let $x, y \in \bar{A}, 0 \leq \lambda \leq 1 \Rightarrow \exists a, b \in A$ such that $\mu(x-a)<r, \mu(x-b)<r$

Since $A$ is convex $\Rightarrow \alpha a+(1-\alpha) b \in A$

$\alpha x+(1-\alpha) y-(\alpha a+(1-\alpha) b)$

$=\alpha(x-a)+(1-\alpha)(y-b)$

$\mu(\alpha x+(1-\alpha) y-(\alpha a+(1-\alpha) b))$

$\leq \alpha \mu(x-a)+(1-\alpha) \mu(y-b)$

$<\alpha r+(1-\alpha) r=r$

$\Rightarrow \alpha x+(1-\alpha) y \in \bar{A} \Rightarrow \bar{A}$ is convex set.

\section{Lemma (1.26):}

Let $(X, \mu, *)$ be afuzzy modular space and $A \subset X$, if for any $x \in \bar{A}$, then there exist a sequence $\left\{x_{\mathrm{n}}\right\}$ in $A$ such that $\lim _{\mathrm{n} \rightarrow \infty} \mu\left(x_{n}, \mathrm{x}, \mathrm{t}\right)=1$ for all $t>0$.

\section{Proof:}

Suppose $x \in \bar{A}$

Since $\bar{A}=A \cup A \Longrightarrow x \in A$ and $x \in A$

If $x \in A$, the $\{x, x, \ldots \ldots\} \rightarrow x$

If $x \in A^{\prime}$ and $x \notin A \Rightarrow \forall n \in Z^{+}, r=\frac{1}{n}$,

$$
\begin{array}{r}
B(x, r, t)-\{x\} \cap A \neq \emptyset \\
x_{n} \in B(x, r, t) \cap A \Rightarrow x_{n} \in A \in A \\
\Rightarrow \mu\left(x_{n}-x, t\right)>1-\frac{1}{n}
\end{array}
$$

If $x \in A$

Let $G$ be an open set contain

$$
X \Rightarrow \exists r>0, B(x, r, t) \subseteq G
$$

Since $\left\{x_{n}\right\}$ in $A$ such that $x_{n} \rightarrow x$ as $n \rightarrow \infty$

$$
\begin{aligned}
\Rightarrow \mu\left(x_{n}-x, t\right) & >1-r \\
\text { Then } x_{n} & \in G \Longrightarrow G-\{x\} \cap A \neq \emptyset
\end{aligned}
$$

Then $x \in \bar{A}$.

Definition (1.27):
A fuzzy modular space $(X, \mu, *)$ is
called a locally convex if there is a local base $\beta$ at 0 in $X$ such that every member of $\beta$ are convex sets.

\section{Example (1.28) :}

Every fuzzy modular space is locally convex

Solution: Let $(X, \mu, *)$ be afuzzy modular space Let $\beta=\{B(r, 0, t): r>0\}$, where $B(r, 0, t)=\{x \in X: \mu(x, t)>1-r\}$, Let $G$ be an open set in $X$,then $G$ is the union of open balls , so $0 \in B(r, 0, t) \subset G$ for some $r>0$, then $\beta$ is alocal base at $0 \operatorname{in} X$

Since every open ball is convex set, then $B(x, 0, t)$ is convex set for all $r>0$, then $\beta$ is a convex local base at 0 in $X$ Therefore $(X, \mu, *)$ is locally convex space. 


\section{References:}

References

\section{References :}

[1] S.S.A bed, , K.A. Abdul Sada," An

Extension of Brosowski - Meinaraus Theorem in Modular Spaces" ,Inter. J. of Math .Anal . Hikari Ltd ., 11,18,877-882, (2017).

[2] B. Schweize and A. Sklar, "Statistical metric spaces ," Pacific Journal of Mathematics ,Vol.10, pp.313-334,(1960).

[3] E. Kreyszing, Introductory functional analysis with application, John Wiley and Sons , Inc .,(1978) .

[4] J. B. James and Esfandiar Eslami,
Noori .F/Al-ham .S

An Introduction to Fuzzy Logic and Fuzzy Sets ,New York:Physica-Verlag,(2002).

[5] A .George and P .Veeramani ," Fuzzy Set and Systems, vol.64,no.3, pp.395-399,(1994). [6] S.Yonghong and Wei Chen ,“ On Fuzzy Modular Spaces," School of Mathematics, Beijing 100081,China ,(2013) .

[7] L.A. Zadeh Fuzzy Sets, Info[1] A. George and P.Veeramani ,"On some result in fuzzy metric rmation and control ,8:338-353,(1965). 
بعض خصائص فضاء الوحدات الضبابي التبولوجي

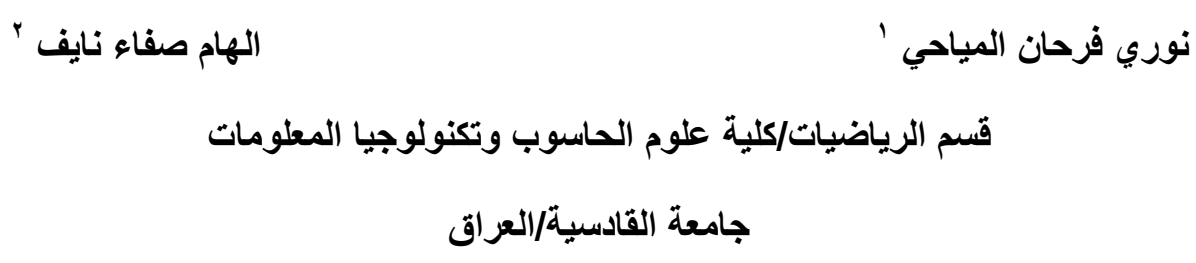

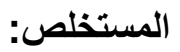

في هذا البحث ، عرفنا فضاء الوحدات الضبابي التبولوجي والمفاهيم المتعلقة به مثل الكرات المفتوحة والمغلقة ،المجموعة المثاء المحدبة ،التحدب المحلي ،المجموعة المفتوحة والمغلقة .وبرهنا بعض النتائج المتعلقة بهذا الفضاء . 